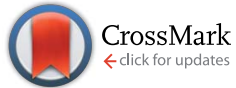

Cite this: RSC Adv., 2014, 4, 32628

\title{
In vitro evaluation of copper-chelating properties of flavonoids $\uparrow$
}

Michal Říha, ${ }^{a}$ Jana Karlíčková, ${ }^{\mathrm{b}}$ Tomáš Filipský, ${ }^{a}$ Kateřina Macáková, ${ }^{\mathrm{b}}$ Liliana Rocha, ${ }^{\mathrm{ac}}$ Paolo Bovicelli, ${ }^{d}$ Ilaria Proietti Silvestri, ${ }^{e}$ Luciano Saso, ${ }^{f}$ Luděk Jahodáŕ, ${ }^{b}$

Radomír Hrdina ${ }^{a}$ and Přemysl Mladěnka*a

Copper is an essential trace element involved in plenty of redox reactions in living systems, however, unbound copper ions cause damage to various biomolecules via excessive generation of reactive oxygen species. Flavonoids, ubiquitous plant secondary metabolites, possess complex effects on human health and chelation of transient metal ions is one of their proposed mechanisms of action. In this in vitro study, 26 flavonoids from various subclasses were screened for their interactions with both copper oxidation states at four (patho)physiologically relevant $\mathrm{pH}$ conditions (4.5, 5.5, 6.8 and 7.5) by two spectrophotometric approaches and compared with the clinically used copper chelator trientine. In a slightly competitive environment, the majority of flavonoids were able to chelate cupric ions, however, under more competitive conditions, only flavones and flavonols were able to chelate both cupric and cuprous ions. Apparently, the 2,3-double bond was essential for stable copper chelation. The most efficient copper chelation sites were the 3-hydroxy-4-keto group in flavonols and the 5,6,7-trihydroxyl group in flavones. On the other hand, the $3^{\prime}, 4^{\prime}$-dihydroxyl group was associated only with a weak activity. 3-Hydroxyflavone, kaempferol and partly baicalein were even more potent than trientine in the acidic environment, however, none of the tested flavonoids was able to surpass it at physiological $\mathrm{pH}$ or slightly acidic conditions. In conclusion, flavonoids possessing appropriate structural characteristics were efficient copper chelators and some of them were even more potent than trientine under acidic conditions.

\section{Introduction}

Flavonoids represent a large group of polyphenolic compounds which belong to plant secondary metabolites. Their ubiquity in the plant kingdom and high content especially in fruits and vegetables make them a common component of the human diet. Flavonoids are 2-phenyl-1-benzopyran-4-one derivatives

${ }^{a}$ Department of Pharmacology and Toxicology, Faculty of Pharmacy in Hradec Králové, Charles University in Prague, Heyrovského 1203, 50005 Hradec Králové, Czech Republic. E-mail: mladenkap@faf.cuni.cz; Fax: +420 495067170; Tel: +420 495067295

${ }^{b}$ Department of Pharmaceutical Botany and Ecology, Faculty of Pharmacy in Hradec Králové, Charles University in Prague, Heyrovského 1203, 50005 Hradec Králové, Czech Republic

${ }^{c}$ ERASMUS student at ${ }^{a}$; home institution: Faculty of Pharmacy, University of Porto, Praça Gomes Teixeira, 4099-002 Porto, Portugal

${ }^{d}$ Department of Chemistry, Institute of Biology, Molecular Medicine and NanoBiotechnologies (IBMN), Sapienza University of Rome, Piazzale Aldo Moro 5, 00185 Rome, Italy

${ }^{e}$ Department of Chemistry, Sapienza University of Rome, Piazzale Aldo Moro 5, 00185 Rome, Italy

${ }^{f}$ Department of Physiology and Pharmacology "Vittorio Erspamer”, Sapienza University of Rome, Italy

$\dagger$ Electronic supplementary information (ESI) available. See DOI: $10.1039 / \mathrm{c} 4 \mathrm{ra} 04575 \mathrm{k}$ which can be classified into several subclasses according to their basic chemical structure: flavones, flavonols, flavanones, flavanonols, and flavanols. Plenty of in vitro, in vivo and several epidemiological studies have suggested their health-promoting effects. Miscellaneous properties including antioxidant, antimicrobial, anti-inflammatory, anticancer, vasoactive or hepatoprotective may be responsible for this. ${ }^{1-3}$ The former theories explaining the effects only by direct scavenging of reactive oxygen species (ROS) seem to be overcome because flavonoids are able to chelate transient metals (particularly iron and/or copper), to block several enzymes producing ROS, and to specifically interact with other targets (direct antiaggregatory and vasodilatory potential) as well. ${ }^{4}$ While the interactions of flavonoids with iron, enzymes and specific targets have been extensively studied (reviewed in ref. 4), data on interactions of flavonoids with copper are rather sparse. Quercetin and rutin were mostly examined and only a few other compounds have been tested..$^{5-10}$ As far as we know, no complex comparison of copper-chelating properties of flavonoids has been published yet.

Copper is an essential trace metal whose function in living systems is based predominantly on its ability to change between two oxidation states, a cuprous and a cupric one. Cuproenzymes are involved in many vital processes such as cell respiration, 
ROS elimination, biosynthesis of neurotransmitters and connective tissue. ${ }^{\mathbf{1 1}}$ The fate of copper in the human body is complex and has not been fully understood. However, a meticulous regulation of copper homeostasis is necessary because unbound copper ions participate in the formation of ROS due to the catalysis of the Fenton-like reaction which consequently causes damage to various biological structures. ${ }^{\mathbf{1 2}}$ A typical example of copper homeostasis disorder is Wilson's disease, which is caused by the chronic copper overload due to a specific genetic abnormality. ${ }^{13}$ Despite some controversy, the disruption of copper homeostasis is involved in various pathological states including neurodegenerative diseases, tumours and acute myocardial infarction. ${ }^{\mathbf{1 4 - 1 7}}$

The chelation approach is promising in pathological states associated with the excess or dysregulation of transition metals, however, the clinical use of copper chelators is currently restricted only for the treatment of Wilson's disease. On the other hand, the use of copper-chelating agents is absolutely essential in this case: until the effective pharmacological treatment based on D-penicillamine was available, Wilson's disease was fatal. The research of copper chelators has not been so successful up to now since currently only two other copper chelators, trientine and tetrathiomolybdate, are used in the treatment of Wilson's disease. ${ }^{13}$ Interestingly, although D-penicillamine is considered as a standard copper chelator in the clinical practice, it is a much less potent copper chelator than trientine. ${ }^{18}$ The research of novel copper chelators for the other above mentioned disorders, in particular that of the central nervous system, is ongoing but most compounds are still in the phase of experimental testing.

When screening metal chelators, several other factors should be considered, e.g. lipophilicity of the parent compound and the complex, affinity for the both cupric and cuprous ions, participation of the complex in redox chemistry, influence by $\mathrm{pH}$ because lower than physiological $\mathrm{pH}$ are characteristic for both physiological conditions (copper absorption in the duodenum) and several pathologies (tumours, acute myocardial infarction and inflammation), and the last but not the least the potential toxicity of the compound.

Therefore this study was aimed at a detailed in vitro evaluation of copper-chelating properties of various flavonoids which are generally considered as non-toxic. Their chelation efficiency was assessed at four $\mathrm{pH}$ conditions ranging from 4.5 to 7.5, towards both cupric and cuprous ions, and was compared with trientine, a clinically used copper chelator. The emphasis was given especially on the elucidation of the structure-activity relationship. Additionally, the relative stability of the formed complexes was assessed.

\section{Materials and methods}

\section{Reagents and solutions}

Stock solutions of cupric ions (cupric sulphate pentahydrate, $\mathrm{CuSO}_{4} \cdot 5 \mathrm{H}_{2} \mathrm{O}$ ) and cuprous ions (cuprous chloride, $\mathrm{CuCl}$ ) were prepared in water (Milli-Q RG, Merck Millipore, Massachusetts, U.S.A.) or in the aqueous solution of $0.1 \mathrm{M} \mathrm{HCl}$ and $1 \mathrm{M} \mathrm{NaCl}$, respectively. The corresponding fresh working solutions $(0.25$
$\mathrm{mM}$ ) were prepared by dilution in DMSO (BCS method) or distilled water (hematoxylin method). Hydroxylamine hydrochloride and bathocuproinedisulfonic acid disodium salt (BCS) were dissolved in distilled water. Hematoxylin was dissolved in DMSO and its working solution $(0.25 \mathrm{mM})$ was usable for no longer than $90 \mathrm{~min}$. All flavonoids were dissolved in DMSO, while trientine in distilled water.

Experiments were performed in $15 \mathrm{mM}$ buffers, acetate $(\mathrm{pH}$ 4.5 and 5.5) and HEPES (pH 6.8 and 7.5).

Luteolin-4'-O-glucoside was purchased from Extrasynthese (France). Mosloflavone and negletein were synthesized by a convergent synthesis starting from chrysin according to the previous report. ${ }^{19}$ All other chemicals were purchased from Sigma-Aldrich (Germany).

\section{Copper chelation assessment}

Two spectrophotometric methods were used for the screening of copper-chelating properties: an assay based on BCS and a complementary assay using hematoxylin. These methods were previously reported in the details. ${ }^{18}$

The hematoxylin assay: different concentrations of a tested compound were mixed for 2 min with cupric ions in the presence of a buffer. Non-reacted cupric ions were mixed for next 3 min with the indicator hematoxylin and the absorbance was measured thereafter and after other $4 \mathrm{~min}$. Different wavelengths were used according to $\mathrm{pH}$ : $595 \mathrm{~nm}(\mathrm{pH}$ 5.5), $590 \mathrm{~nm}$ ( $\mathrm{pH}$ 6.8), and $610 \mathrm{~nm}(\mathrm{pH} 7.5)$, as reported earlier. ${ }^{18}$

A similar approach was used in the BCS assay: different concentrations of a tested compound were mixed with cupric or cuprous ions in an excess of buffer and mixed for $2 \mathrm{~min}$. In the case of cuprous ions, hydroxylamine was added before the copper solution in order to retain copper in its reduced state. In the case of cupric ions, hydroxylamine was added after mixing by virtue of reduction of non-reacted cupric ions. The nonchelated copper was then evidenced in both cases by the indicator BCS and absorbance was read immediately and after 5 $\min$ at $484 \mathrm{~nm}$.

All experiments were performed in 96-well microplates, at least in duplicates, at room temperature. A Synergy HT MultiDetection Microplate Reader (BioTec Instruments, Inc., USA) was used for all spectrophotometric measurements.

\section{Statistical analysis}

The amount of remaining copper was calculated from the difference of absorbance between the tested sample (with the indicator) and its corresponding blank (without indicator) divided by the difference of the control sample (the known amount of copper without the tested substance) and its control blank.

The dose-dependent curves with 95\% confidence (prediction) intervals were constructed by GraphPad Prism 6 for Windows (GraphPad Software, USA). Each curve of an efficient substance was composed from at least four points: first minimal point was approximately $0-5 \%$ chelation, last point was maximal chelation. Maximal chelation was considered 95$100 \%$ for high-affinity chelation or lower in cases when plateau bellow this value was found. The efficiency of the copper 
chelation was calculated at the concentration ratio $1: 1$ and $10: 1$, substance : copper, respectively, according to the curve equations:

$$
y=\max /\left(1+10 \exp \left(\left(\log \mathrm{ER}_{50}-x\right) \times k\right)\right)
$$

where $y$ is the amount of chelated copper ions in per cent; max the maximal chelation of copper ions in per cent; $\mathrm{ER}_{50}$ the effective substance: copper concentration ratio needed to chelate $50 \%$ of the copper; $x$ the common logarithm of the substance : copper concentration ratio; and $k$ the slope of the curve.

In the hematoxylin assay, $\mathrm{ER}_{50}$ were used for the comparison of the chelation potencies. The corresponding SD values were computed from the relation between confidence intervals and $\mathrm{SD}^{20}$

Because the used indicators act virtually as copper chelators, the competition between the tested compound and the indicator occurs. When the compound forms unstable complexes, it is possible to assess the relative stability of the substance : copper complex. The value of relative stability $s$ was calculated as follows:

$$
s=y_{2} / y_{0}
$$

where $y_{2}$ is the percentage of chelated copper in the second measurement (after 4 or $5 \mathrm{~min}$ in the hematoxylin or BCS methodology, respectively), and $y_{0}$ is the amount of chelated copper ions at first measurement.

Data are expressed as mean \pm SD. Differences were considered significant at $p<0.05$, unless stated otherwise. The differences in chelation and relative stability of copper complexes were performed by one-way ANOVA test followed by Bonferroni's multiple comparisons test.

\section{Results}

Firstly, we assessed copper-chelating properties of all 26 flavonoids (for chemical structures see Fig. S1†) by the use of the hematoxylin assay because the methodology is less competitive than that of BCS and therefore less efficient copper chelators may be revealed as well. ${ }^{18}$

\section{Hematoxylin assay}

All tested flavonoids excluding non-substituted flavone and 7hydroxyflavone were able to chelate copper in this complementary assay (Fig. 1). Although this methodology is not principally aimed at the determination of the stoichiometry, the stoichiometry could be assessed in the case of potent copper chelators. E.g., if $\mathrm{ER}_{50}$ equals to 1, this mean that at the ratio $1: 1$ about $50 \%$ of copper was chelated, thus at the ratio $2: 1$, flavonoid to copper, respectively, about $100 \%$ of copper will be chelated and therefore the stoichiometry of the complex would be $2: 1$. Analogously, $\mathrm{ER}_{50}$ of 0.25 suggests the stoichiometry of $1: 2$ and 0.5 that of $1: 1$. In the case of low-affinity chelators, the $\mathrm{ER}_{50}$ is apparently higher to fit in a possible chelation stoichiometry and thus the stoichiometry cannot be assessed in such cases.

Flavones luteolin and baicalein and flavonol myricetin were the most potent compounds. They were more active than trientine and their $\mathrm{ER}_{50}$ values were approximately 0.25 suggesting the complex stoichiometry $1: 2$ (flavonoid : copper). Trientine is well known to form a complex $1: 1$ stoichiometry and herein the expected $\mathrm{ER}_{50}$ of 0.5 was confirmed. In contrast to trientine which formed the same complex at all $\mathrm{pH}$ conditions, luteolin formed likely the complex $1: 1$ at $\mathrm{pH} 5.5$ while the stoichiometry of complexes with baicalein and myricetin was on average between $1: 1$ and $1: 2$ at this $\mathrm{pH}$ and thus it could not be established with certainty from the current experiment. Quercetin formed likely complexes with stoichiometry $1: 2$ at pH 6.8 as well but such complexes were not stable at $\mathrm{pH} 7.5$ and 5.5. At the latter $\mathrm{pH}$, apparently $1: 1$ stoichiometry was observed.

The $\mathrm{ER}_{50}$ around 0.5 was observed in the case of negletein and baicalin at $\mathrm{pH} 6.8$ and 7.5 and in rutin at $\mathrm{pH} 6.8$ suggesting that these flavonoids are able to form complexes with stoichiometry $1: 1$. Again, their affinity for cupric ions at $\mathrm{pH} 5.5$ was lower in comparison to slightly acidic or physiological $\mathrm{pH}$ conditions.

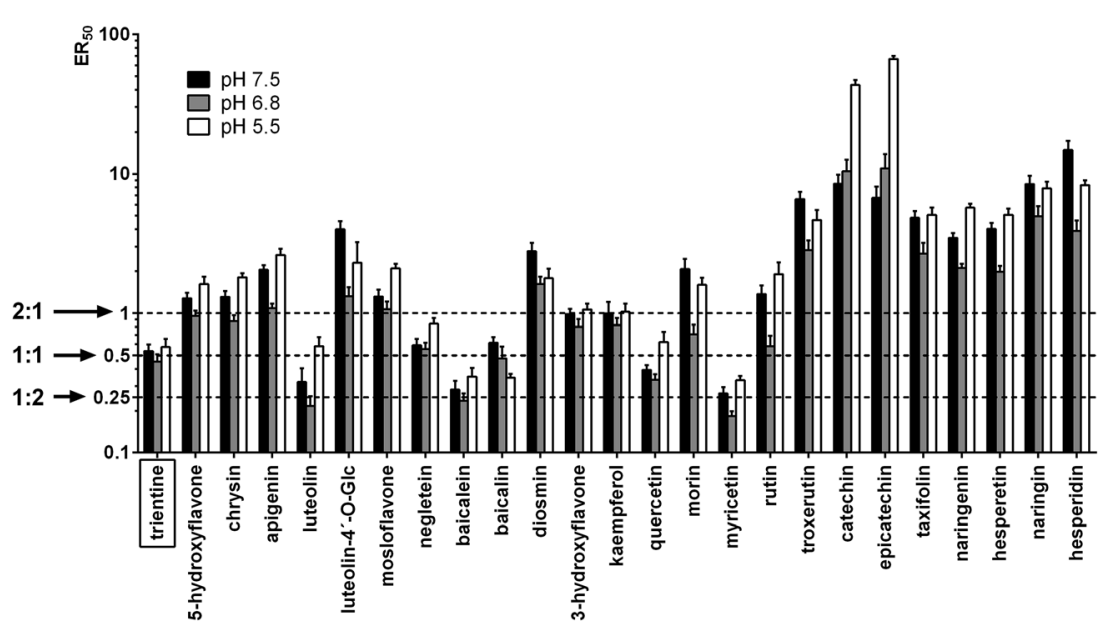

Fig. 1 The chelation of cupric ions by flavonoids and trientine according to the hematoxylin assay. Data are expressed as ER $\mathrm{R}_{50}$ values. 
The $\mathrm{ER}_{50}$ of 1 , implicating stoichiometry $2: 1$, was observed in the case of flavonols without the catechol ring B (3-hydroxyflavone and kaempferol) at all tested $\mathrm{pH}$ conditions. Flavones without the catechol ring B including 5-hydroxyflavone, chrysin, apigenin, mosloflavone and luteolin- $4^{\prime}-O$-glucoside formed the complexes with cupric ions at $\mathrm{pH} 6.8$ with stoichiometry $2: 1$.

In the cases of flavanones and flavanols, the stoichiometry cannot be established because these flavonoids have high $\mathrm{ER}_{50}$ indicating their lower affinity to copper.

The active flavonoids formed stable complexes at $\mathrm{pH} 5.5$ and 6.8, however at $\mathrm{pH} 7.5$, the relative stability depended on the ratio of the flavonoid to copper ions and it dropped with decreasing ratio (Fig. $\mathrm{S} 2 \dagger$ ). The exceptions were baicalein, 3- hydroxyflavone and luteolin which formed the stable complexes even at $\mathrm{pH} 7.5$.

\section{Structure-activity relationship}

The 5,6,7-trihydroxyl or 6,7-dihydroxyl; 3',4'-dihydroxyl; 3hydroxyl-4-keto or 5-hydroxy-4-keto functional groups were associated with cupric chelation. In order to highlight the relationships between structural modifications and the activity, following schemes (Fig. 2-4) were prepared.

In the hematoxylin methodology, the chelating groups were evidently responsible for a majority of the differences in copper chelation, e.g.:

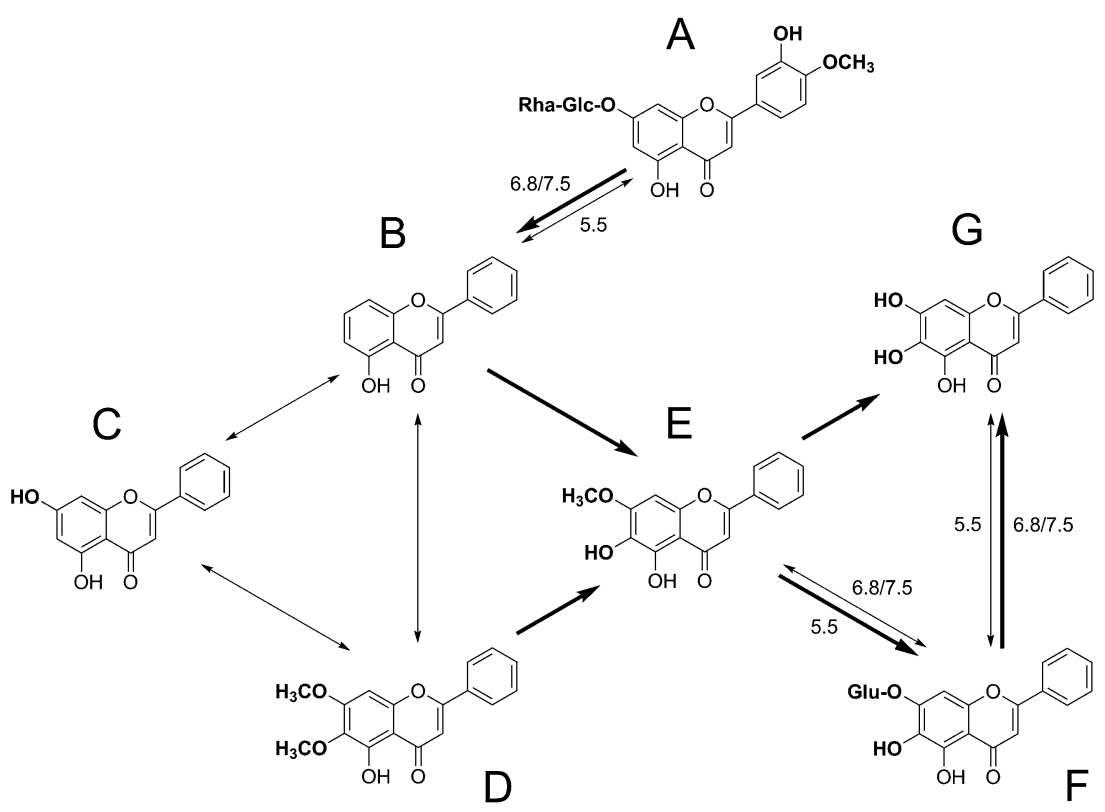

Fig. 2 The effect of different substitution in ring A in flavones. (A) Diosmin, (B) 5-hydroxyflavone, (C) chrysin, (D) mosloflavone, (E) negletein, (F) baicalin, and $(G)$ baicalein. The direction of an arrow shows the relationship between compounds: a bold one-way arrow means a significant difference $(p<0.05)$, a two-way arrow the same potency. Glc: glucose, Glu: glucuronic acid, Rha: rhamnose.

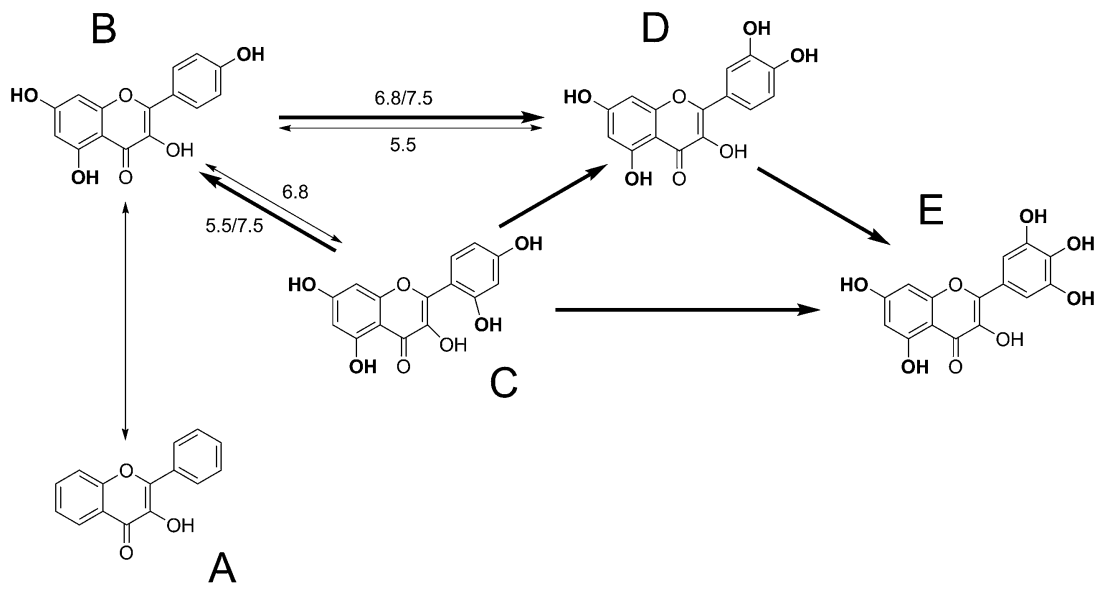

Fig. 3 The effect of a hydroxyl substitution in flavonols. (A) 3-Hydroxyflavone, (B) kaempferol, (C) morin, (D) quercetin, and (E) myricetin. The direction of an arrow shows the relationship between compounds: a bold one-way arrow means a significant difference $(p<0.05)$, a two-way arrow the same potency. 


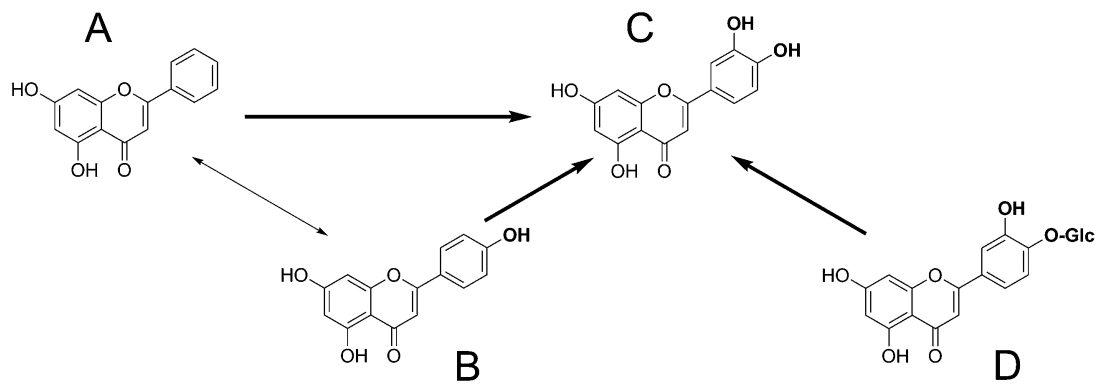

Fig. 4 The effect of the substitution in ring B in flavones. (A) Chrysin, (B) apigenin, (C) luteolin, and (D) luteolin-4'-O-glucoside. The direction of an arrow shows the relationship between compounds: a bold one-way arrow means a significant difference $(p<0.05)$, a two-way arrow the same potency.

- three adjacent hydroxyl groups in ring A or in ring B were associated with better copper chelation than two adjacent hydroxyl groups (Fig. 2 and 3);

- the catechol moiety in ring B increased the chelation in comparison with an isolated hydroxyl group, in particular at $\mathrm{pH}$ 6.8 and 7.5 (Fig. 3 and 4);

- the 3-hydroxy-4-keto chelation site apparently improved copper chelation as the blockade of the 3-hydroxyl group decreased the copper chelation potential (quercetin was more potent than rutin and troxerutin). The effect of that chelation site was confirmed even in the absence of the 2,3-double bond: flavanols with the catechol ring $\mathrm{B}$ were less potent than taxifolin possessing both catechol ring B and the 4-keto group under acidic conditions (Fig. 1);

- similarly, the 5-hydroxy-4-keto chelation site was associated with the copper chelation activity (e.g. non-chelating flavone $v s$ chelating 5-hydroxyflavone).

The non-chelating groups influenced only slightly the resulting chelation effect with few exceptions:

- the adjacent hydroxyl group in the position $2^{\prime}$ decreased the chelation potential of the 3-hydroxy-4-keto chelation site (Fig. 3C vs. 3D);

- in flavanones, a sugar in the position 7 significantly reduced the copper chelation potential (Fig. 1), similarly, a sugar in the position 7 in flavones decreased the chelation potential of the 5-hydroxy-4-keto chelation site (Fig. $2 \mathrm{~F}$ vs. $2 \mathrm{G}$ );

- contrarily, a sugar in the position 7 was more advantageous than a methoxyl group in this position at least at pH 5.5 (Fig. 2F vs. 2E).

\section{BCS assay}

In this assay, all 24 flavonoids which chelated copper ions in the hematoxylin method were tested. In contrast to the former assay, only the members of flavones and flavonols were able to substantially chelate copper in this competitive approach including BCS. This suggested that the $3^{\prime}, 4^{\prime}$-dihydroxyl group alone was insufficient for copper chelation in the competitive conditions in contrast to the previously mentioned slightly competitive hematoxylin approach. A blocking of the catechol group in the case of luteolin- $4^{\prime}$-O-glucoside or diosmin abolished absolutely the remaining activity. For an overview, the cupric chelation ability of active flavonoids was compared at the ratios of
$1: 1$ and $10: 1$ (flavonoid : copper) (Fig. 5). All tested compounds showed generally higher activity towards cupric ions, but the results are in general analogous for cuprous ions (Fig. S3†).

In most cases, the chelation ability significantly dropped with increasing acidity of the environment and the majority of flavonoids chelated hardly any or no copper ions at $\mathrm{pH}$ 4.5. Interestingly, in contrast to the hematoxylin method or the chelation curves of trientine, the majority of tested flavonoids were not able to chelate $100 \%$ of the copper even at very high concentration ratios (the representative examples are shown in Fig. S4†). Rather a plateau was observed in majority of flavonoids. This is apparent from the Fig. 5 where the copper chelation of 3-hydroxyflavone or kaempferol increased from the ratio of $1: 1$ to $10: 1$ while baicalein mostly reached its maximal chelation approximately at the ratio of $1: 1$.

None of the tested flavonoids was more potent than trientine at the physiological or slightly acidic conditions even if 3hydroxyflavone and partly as well kaempferol were similarly active at the ratio of $10: 1$. However, under the acidic conditions, the both mentioned flavonols were more potent than trientine at the ratio of $10: 1$ for chelation of the both cupric and cuprous ions. Baicalein, due to its inability to chelate $100 \%$ of copper, surpassed trientine only at $\mathrm{pH} 4.5\left(\mathrm{Cu}^{2+}\right.$ at the ratio of $1: 1, \mathrm{Cu}^{+}$at the ratio of $10: 1$ ) while at pH 5.5 it was more active chelator of cuprous ions and non-inferior chelator of cupric ions. All other tested flavonoids were less efficient than trientine at all conditions.

An additional analysis was performed with active copper chelators, i.e. those with chelation higher than $30 \%$ at the ratio of $10: 1$ (Fig. S5 ). The relative stability of flavonoids was independent on $\mathrm{pH}$, the only exception was baicalein which formed less stable complexes at more acidic conditions. 3Hydroxyflavone, kaempferol, quercetin and myricetin formed stable complexes at all tested $\mathrm{pH}$ conditions with the relative stability comparable with complexes of trientine at $\mathrm{pH}$ 6.8/7.5 (Fig. S5 $\dagger$ ). On the other hand, the complexes of other assessed flavones were significantly less stable than in the case of trientine. The described phenomenon was independent on the oxidation state of copper ions.

\section{Structure-activity relationship}

In contrast to the hematoxylin methodology, the impact of different substitutions was more complex and markedly 
A
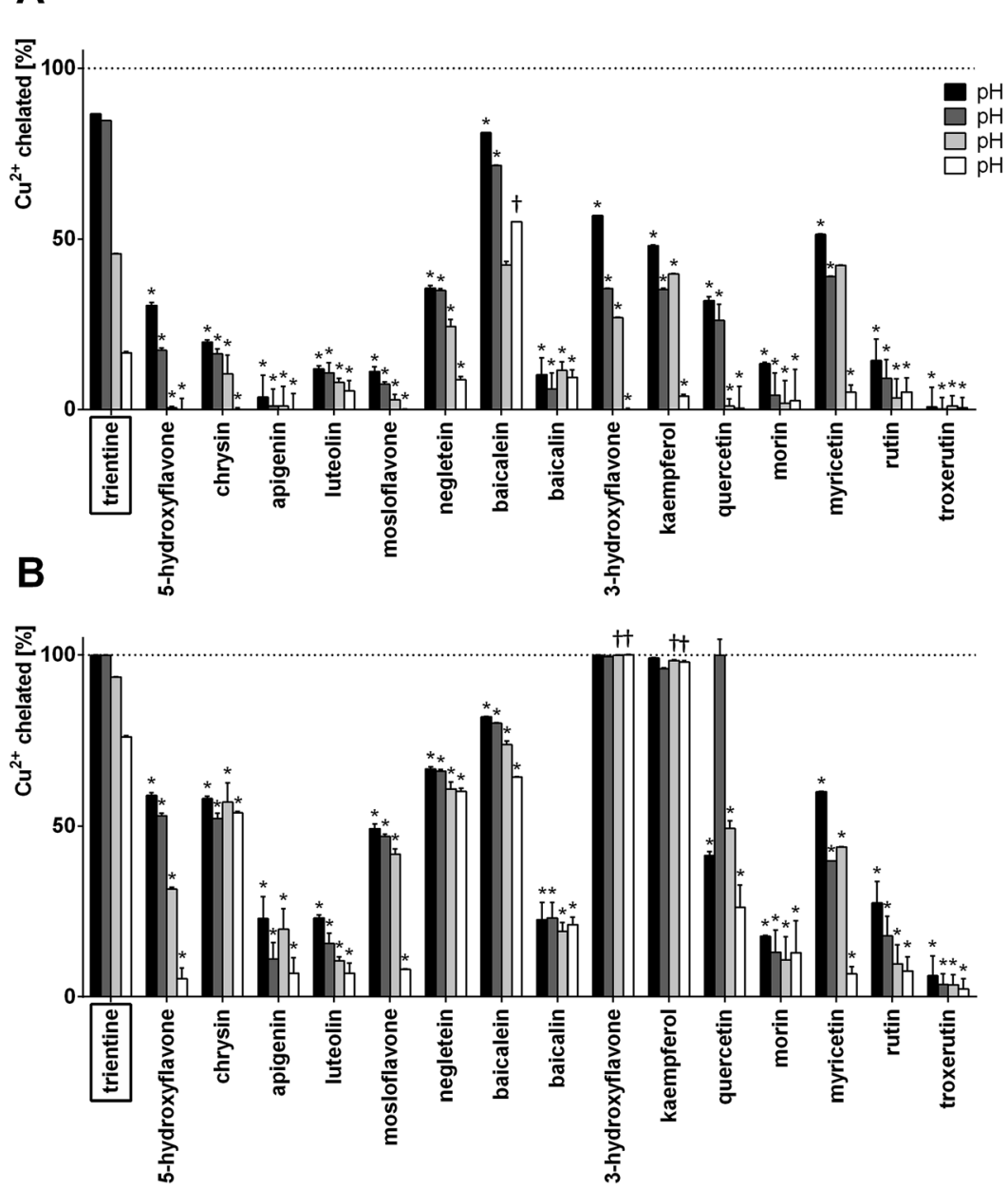

Fig. 5 The chelation of cupric ions by flavonoids in comparison with trientine, at the ratios of $1: 1$ (A) and $10: 1$ (B) (flavonoid : copper). * Less potent than trientine, $\uparrow$ more potent than trientine $(p<0.05)$.

dependent on copper oxidation state and pH (Fig. 6-9). The most important findings are:

- a substitution of the free 3-hydroxyl group was associated with a marked decrease in the chelation activity (Fig. 6);
- a presence of adjacent hydroxyl groups in ring A increased the chelation potency while a methoxyl group or a sugar had negative influence on it (Fig. 7). On the contrary, a hydroxyl substitution in ring $\mathrm{B}$ had no influence (Fig. 8A vs. 8B) or even deteriorated the activity (Fig. 8 and 9);

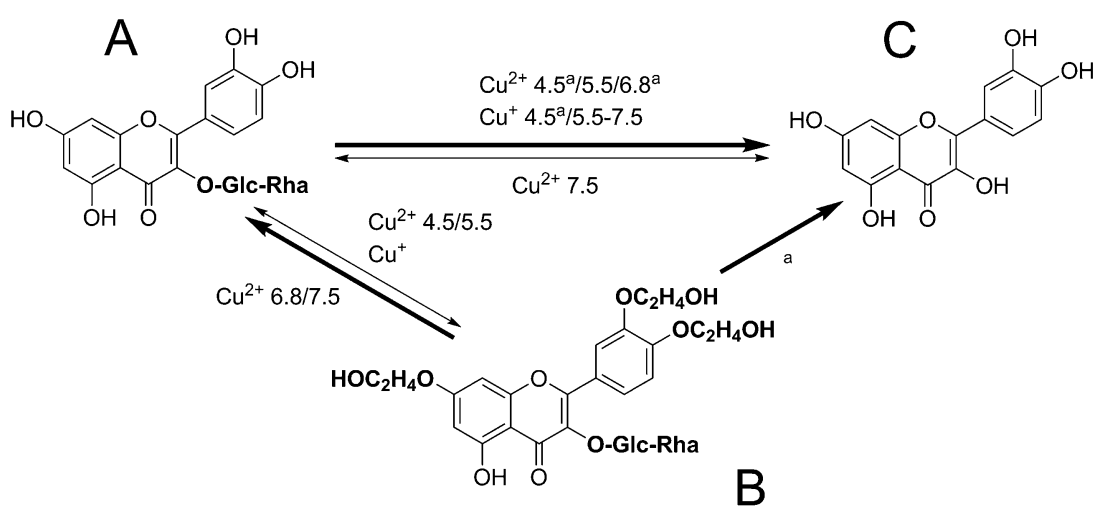

Fig. 6 The effect of the 3-hydroxyl group and additional hydroxyl groups in flavonols. (A) Rutin, (B) troxerutin, (C) quercetin, a significance was found for $\mathrm{pH} 4.5$ and cuprous ions at the ratios higher than $10: 1$ (flavonoid : copper, respectively). The direction of an arrow shows the relationship between compounds: a bold one-way arrow means a significant difference $(p<0.05)$, a two-way arrow the same potency. Glc: glucose, Rha: rhamnose. 


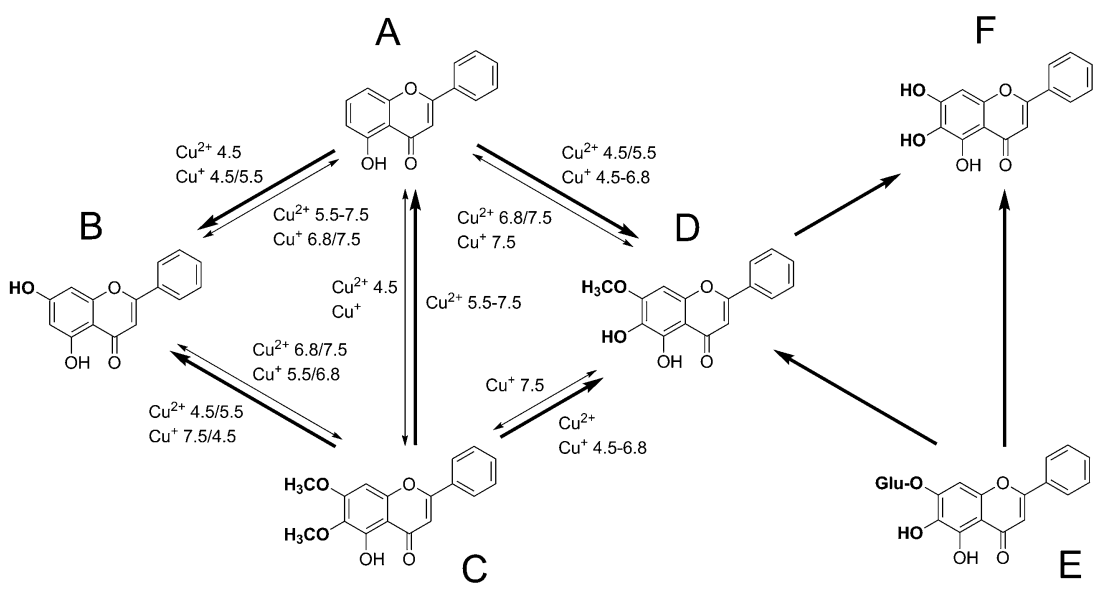

Fig. 7 The effect of different substitution in ring A in flavones. (A) 5-Hydroxyflavone, (B) chrysin, (C) mosloflavone, (D) negletein, (E) baicalin, and (F) baicalein. The direction of an arrow shows the relationship between compounds: a bold one-way arrow means a significant difference $(p<$ 0.05), a two-way arrow the same potency. Glu: glucuronic acid.

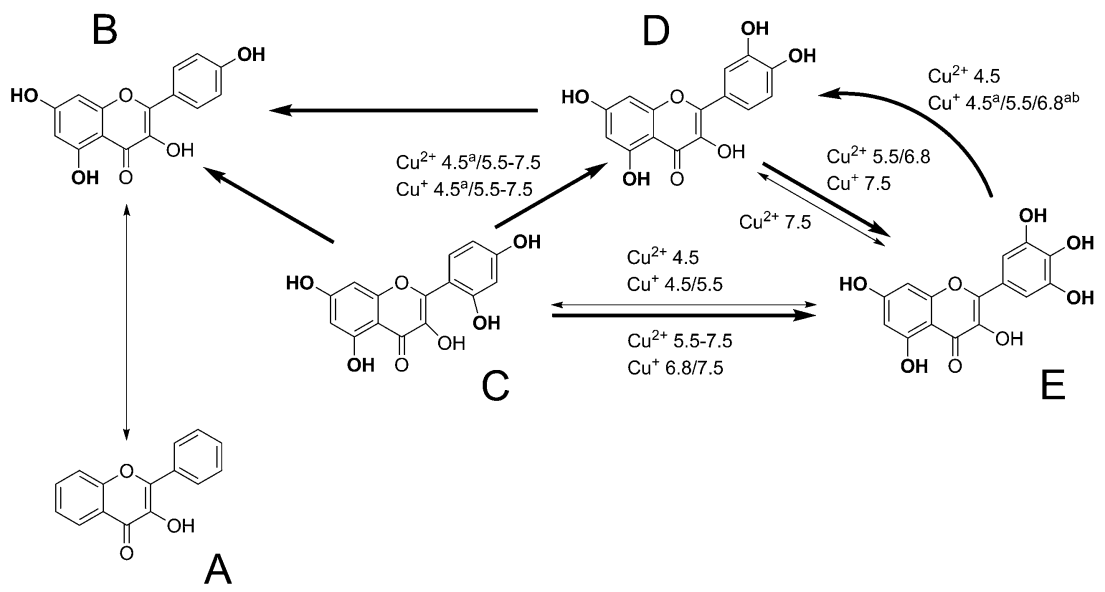

Fig. 8 The effect of hydroxyl substitution in flavonols. (A) 3-Hydroxyflavone, (B) kaempferol, (C) morin, (D) quercetin, (E) myricetin, ${ }^{a}$ significance was found at the ratios higher than $10: 1$ (flavonoid : copper), ${ }^{b}$ at the ratios lower than $4: 1$ (flavonoid : copper) myricetin was more potent than quercetin. The direction of an arrow shows the relationship between compounds: a bold one-way arrow means a significant difference ( $p<$ 0.05), a two-way arrow the same potency.

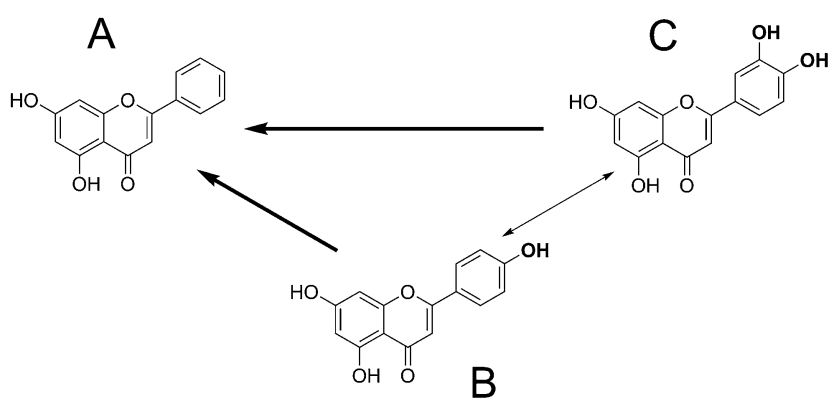

Fig. 9 The effect of substitution in ring B in flavones. (A) Chrysin, (B) apigenin, and (C) luteolin. The direction of an arrow shows the relationship between compounds: a bold one-way arrow means a significant difference $(p<0.05)$, a two-way arrow the same potency.
- a hydroxyl group in the position $2^{\prime}$ weakened the effect of the essential 3-hydroxy-4-keto chelation site (Fig. 9C vs. 9B).

\section{Discussion}

While flavonoids-iron interactions have been studied in a detailed way, ${ }^{21-30}$ such attention has not been focused on copper. This study demonstrated that flavonoids across different subclasses were able to chelate copper ions, however, in a very variable manner. The chelating sites for copper does not substantially differ from that of iron or other metals. ${ }^{22,31}$ In harmony with published data on the both copper and iron chelation, the most important chelation sites were 3-hydroxy-4keto, 5-hydroxy-4-keto, $3^{\prime}, 4^{\prime}$-dihydroxyl (i.e. the catechol ring B), and (5),6,7-(tri)dihydroxyl substitution in ring A. Isolated keto, hydroxyl and methoxyl groups were not associated with metal chelation. 
Contrary to the widespread application of a direct spectrophotometry in the analysis of metal-chelator interactions, ${ }^{32,33}$ we used an indirect spectrophotometric approach. ${ }^{18}$ Contrary to the direct spectrophotometry, a competition between the indicator, which is in fact a strong cuprous chelator BCS, and a tested compound can be useful for the assessment of the formed complex relative stability. While the complementary hematoxylin method is suitable for basic screening of copper chelation ability and suggestion of complex stoichiometry, the assay employing BCS reveals the potency of a chelator.

Although there are important drawbacks of the hematoxylin method including decreasing affinity of hematoxylin for copper with decreasing $\mathrm{pH}$ and resulting impossibility to use the method at $\mathrm{pH} 4.5$, its outcomes concerning suggestion of the stoichiometry appears to be real and in agreement with available literary sources. ${ }^{18}$ Because the affinity of hematoxylin for copper is high at $\mathrm{pH} 7.5$, only very active copper chelators are able to form stable complexes at this $\mathrm{pH}$. This was true for powerful copper chelators of flavonoid class as 3-hydroxyflavone and baicalein. Contrarily, the assessment of copper-flavonoid stoichiometry can be easily measured as well in moderately active chelators at $\mathrm{pH} 6.8$ or $\mathrm{pH} 5.5$ because of lower affinity of hematoxylin for copper at these $\mathrm{pH}$ conditions. This is supported by the fact that all flavonoid-copper complexes were stable at pH 5.5 and 6.8 in contrast to pH 7.5 (Fig. S2 $\dagger$ ).

The stoichiometry of the complexes is apparently based on the number and type of functional groups. At slightly acidic $\mathrm{pH}$ of 6.8, 3-hydroxyflavone and kaempferol with only one possible 3-hydroxy-4-keto chelation site formed the complexes of $2: 1$, flavonoid to copper, similarly to flavones with analogous 5hydroxy-4-keto chelation group (5-hydroxyflavone, chrysin, apigenin, mosloflavone and luteolin- $4^{\prime}$-glycoside). Luteolin and quercetin possessing both (3-hydroxy)-5-hydroxy-4-keto and the catechol ring $\mathrm{B}$ were able to chelate 2 copper atoms per molecule similarly to myricetin equipped with three adjacent hydroxyl groups in ring $\mathrm{B}$ and 3-hydroxy-5-hydroxy-4-keto chelation site. Rutin, although possessing the identical chelation site as luteolin, formed only complexes with $1: 1$ stoichiometry. The likely reason is the steric hindrance of the sugar in the position 3. Specific cases are flavones with 5,6- and 7hydroxyl groups forming complexes of $1: 1$ stoichiometry in case of the presence of only two hydroxyl groups (baicalin and negletein) or chelating 2 molecules of copper per unit in case of the presence of all three hydroxyl groups (baicalein).

By decreasing $\mathrm{pH}$ from 6.8 to 5.5 , a marked drop in the chelation activity of flavonoids with the catechol ring $\mathrm{B}$, the 5hydroxy-4-keto and even the 5,6-dihydroxyl/5,6,7-trihydroxyl chelation sites was observed among all classes of flavonoids. The catechol ring $\mathrm{B}$ is promoted by the majority of researchers as the most important due to its best direct scavenging potential. ${ }^{34,35}$ However, its metal chelating activity is substantial only at physiological $\mathrm{pH}$ and particularly at alkaline conditions $\mathrm{s}^{\mathbf{9} 22,31}$ in contrast to other possible chelation sites, which arises from different $\mathrm{p} K_{\mathrm{a}}$ values of hydroxyl groups in the flavonoid structure. ${ }^{36}$

A similar instability is apparently valid for chelation sites in ring $\mathrm{A}$ or between the rings $\mathrm{A}$ and $\mathrm{C}$. The only $\mathrm{pH}$-independent chelation site was the 3-hydroxy-4-keto site as was clearly demonstrated by 3-hydroxyflavone and kaempferol, whose chelation activity was stable at all $\mathrm{pH}$ including $\mathrm{pH}$ 7.5. Interestingly, myricetin, the only flavonoid tested in this study with three adjacent hydroxyl groups in ring B behaved as well as a $\mathrm{pH}$-independent copper chelator. Sparse data from the literature confirm our findings. Quercetin formed complexes of $1: 1$ or $1: 2$ stoichiometry (flavonoid : copper). A complex of $2: 1$ was not documented by potentiometric and spectrophotometric measurements but may exist likely in a smaller amount at more acidic conditions, as was documented by highly sensitive electrospray ionisation mass spectrophotometry (ESI-MS). ${ }^{7,8,10}$ Rutin is firstly forming complexes with $1: 1$ stoichiometry followed by a disproportionation process leading to $2: 1$ (rutin : copper) complex. $^{7}$ Other groups found complexes of quercetin with copper of $1: 1$ stoichiometry in non-buffered conditions which may be associated with the fact that the catechol ring B may not be available at these conditions in contrast to physiological, slightly basic $\mathrm{pH}$ where deprotonation of catechol hydroxyls is taking place. ${ }^{10}$ It must be mentioned too that the study of de Souza reported complexes of flavonoids with copper in the stoichiometry of $1: 2$ for quercetin, $2: 3$ for rutin and $1: 1$ for 3 hydroxyflavone. The reason for this discrepancy is not known but authors documented identical stoichiometries for flavonoid-iron complexes and those are not in agreement with other studies. ${ }^{22,37}$

Although flavanols and flavanones tested in this study possess the catechol ring B and/or the 5-hydroxy-4-keto or even the 3-hydroxy-4-keto-5-hydroxy chelation site, their copper chelation activity was clearly low and unequivocally lower than that of their close congeners from flavone or flavonol groups. Similarly, spectrophotometric approach did not detect spectral changes in the case of adding cupric ions to flavanones naringenin and naringin at $\mathrm{pH} 5.5$ and 7.4 while the spectrum of flavanol catechin was changed only in the case of $\mathrm{pH} 7.4$. Interestingly, no change was surprisingly observed in the case of taxifolin, ${ }^{9}$ which possesses identical substitution pattern to catechin and 4-keto group in addition, and was shown to be more active than catechin. ${ }^{38}$ In contrast, highly sensitive ESI-MS even at $\mathrm{pH} 3$ found some complexes of catechin and naringenin. ${ }^{8}$ Although authors did not test other mentioned flavonoids, it is apparent, that these flavonoids are able to form only unstable complexes with copper. The reason consists in the lack of the 2,3-double bond, which influences the planarity of the molecule and enables delocalization of $\pi$-electronic system. ${ }^{39}$ It is suggested that copper ions prefer planar or tetrahedral conformation in contrast to octahedral geometries in the case of iron. ${ }^{6}$ This may be relevant, as the stoichiometric complex of $3: 1$, flavonoid to copper, was not observed in this study. Such a complex was found by ESI-MS in low quantities and its formation is clearly less probable., ${ }^{\mathbf{8 , 4}}$

The instability of the catechol ring $\mathrm{B}$ and of the 5-hydroxy-4keto chelation site was further confirmed by the highly competitive BCS methodology. Compounds possessing only the catechol ring $\mathrm{B}$ were almost inefficient while those with the latter were apparently less active than in the hematoxylin method. Such finding was observed earlier in smaller sets of 
flavonoids, where copper was removed from luteolin and rutin complexes after addition of EDTA while only partly from quercetin. ${ }^{\mathbf{5} 10}$ Although, a decrease in the copper chelation potency comparing the hematoxylin and the BCS methodology was observed in chelators with the 3-hydroxy-4-keto group as well, these flavonoids were clearly the most powerful chelators followed by baicalein with three hydroxyl groups in ring A. The importance of the 3-hydroxy-4-keto group is supported by:

(1) mathematical calculations which supposed that the 3hydroxy-4-keto group is the preferred copper chelation site even in the simultaneous presence of the 5-hydroxyl group or the $3^{\prime}, 4^{\prime}$-dihydroxyl group in ring $\mathrm{B}^{\mathbf{4 0}}$

(2) other studies analysing the effect of flavonoids on other metals which confirmed the importance of the 3-hydroxy-4-keto group, although in alkaline media, the catechol might be preferred. $^{22,31}$

Interestingly, the hydroxyl groups in ring B may represent a disadvantage in contrast to the direct scavenging potential. This was demonstrated in the highly competitive approach with BCS. Neither a catechol nor a pyrogallol moiety in ring B improved the chelation. Moreover, these substituents even deteriorated the ability (Fig. 8 and 9). The reason may be paradoxical but important. Probable explanation of this negative effect lies in a reduction of $\mathrm{Cu}^{2+}$ ions, which is substantial by a catechol or a pyrogallol structure in ring B. ${ }^{9}$ Because the affinity for cuprous ions is lower in flavonoids and very high in the case of BCS, flavonoids with highly reducing properties are not able to retain the cuprous ions in the complex structure in competition with the strong indicator BCS. This is in a clear contrast to powerful copper chelators like trientine at $\mathrm{pH} 6.8 / 7.5$ or chloroxine at all tested $\mathrm{pH}$ conditions. ${ }^{18}$ We are currently performing experiments on copper reduction and in line with previous data, ${ }^{9}$ copper reduction is highly dependent on number of hydroxyl groups in ring $\mathrm{B}$ and may therefore influence the copper chelation too.

Similarly, the reduction may explain the failure of baicalein to be the most active copper chelator like in the case of iron, where its affinity for iron was comparable to the standard iron chelator deferoxamine. ${ }^{22}$ Although the chelation ability was growing with the number of free hydroxyl groups in ring A (Fig. 7) and baicalein with the 5,6,7-trihydroxyl groups represented the most potent flavone, it was clearly less efficient than 3-hydroxyflavone. The major difference is that 3-hydroxyflavone or kaempferol were able to chelate $100 \%$ of copper in highly competitive environment with BCS while baicalein did not. The influence of methoxylation of hydroxyl groups in ring A was more pronounced than in the case of iron chelation. On one hand, a methoxyl group instead of the corresponding hydroxyl group abolished the effect of the hydroxyl group which could be observed in the series baicalein $>$ negletein $>$ mosloflavone, but on the other hand mosloflavone possessing the 6,7-dimethoxyl groups was even weaker chelator than the basic flavone, 5hydroxyflavone, under certain conditions. Steric hindrance was probably responsible for this finding. This may be supported by the fact that baicalin with the attached glucose moiety in the position 7 was less potent in comparison with baicalein and even negletein. The 7-hydroxyl group in chrysin favoured the chelation especially under the acidic conditions when compared with 5-hydroxyflavone and mosloflavone.

Although these findings may be relevant to both pharmacology and physiology because flavonoids are common part of our food, the influence of flavonoids on copper pharmacokinetics in human will need additional studies. The fact that 3hydroxyflavone and kaempferol were powerful copper chelators and their effect was under the acidic conditions even more pronounced than that of trientine, a copper chelator used in a clinical practice for the treatment of Wilson's disease, ${ }^{\mathbf{4 1}}$ may be of a clinical relevance. Indeed, trientine is active particularly at neutral and slightly acidic conditions ${ }^{\mathbf{1 8}}$ and because various physiological and pathophysiological aspects (e.g. GIT absorption, tumours, inflamed or ischaemic tissue) influence the acidity of different compartments in the human body, these flavonoids may be possibly advantageous in several pathophysiological situations. However, their pharmacokinetics, which may result in the inactivity, must be taken into account. ${ }^{\mathbf{4 2}}$ None of the tested flavonoids was selective to either oxidation state of copper ions, the majority of them showed higher affinity for the oxidized form. Similar finding was documented in the study with the iron chelation. ${ }^{22}$ But in contrast to the fate of iron in the organism, which is quite well known, the copper pharmacokinetics and in particular the importance of its oxidation state is a subject of debate. ${ }^{43}$ At the moment, even the data on copper absorption are divergent. It appears that the copper transporter CTR1 recognize cuprous ions but the absorption of copper in the form of cupric ions cannot be fully excluded. ${ }^{43}$ Complexes of copper with very active flavonoids 3-hydroxyflavone and kaempferol may lead to either decreased or increased copper absorption depending on possible transport mechanisms and charge. While 3-hydroxyflavone is a synthetic compound and serves as a model molecule, kaempferol is commonly found in plant-derived food and in plants used in traditional medicine ${ }^{\mathbf{4 4}}$ and thus may have potential influence on copper (patho)physiology. On the other hand, other flavonoids with low copper chelation ability together with powerful copper reducing properties may reduce cupric ions present in food into cuprous ones and thus influence the absorption. Some studies analysing these factors are currently being performed in our laboratory. Limited available experimental data in rats give rather equivocal results: feeding with tea or its polyphenols did not affect the absorption of copper, ${ }^{45}$ neither did the administration of rutin and catechin ${ }^{\mathbf{4 6}}$ while in another study, rutin decreased copper content in the liver. ${ }^{47}$ Another interesting factor is the anti- or pro-oxidant activity of copper-flavonoid complexes. A recent study found that copper complexes of flavonoids were more potent antioxidants than non-complexed flavonoids. ${ }^{10}$ However, there is no accordance on this finding in the literature, ${ }^{6}$ and this fact together with possible influence of flavonoids on the copper driven Fenton reaction should be analysed in the future.

\section{Conclusion}

This study demonstrated that the potency of copper chelation is very different among various flavonoids. Although almost all 
flavonoids are able to chelate copper, in the presence of the competitive indicator, their copper chelation potency was rather low in majority of cases. Some compounds, particularly those containing the 3-hydroxyl group in association with the 4-keto group and the 2,3-double bond (3-hydroxyflavone, kaempferol) or possessing the 5,6,7-trihydroxyl substitution (baicalein) were, however, very potent even in highly competitive environment. Their activity in lower $\mathrm{pH}$ conditions was even higher than clinically used copper chelator trientine. On the other hand, the catechol ring $\mathrm{B}$ did not represent significant advantage for the activity, moreover, it may deteriorate the copper chelation under certain conditions, probably through participating in reduction of copper ions. The copper reduction properties must be taken into account for the complex evaluation of flavonoidmetal interactions.

\section{Abbreviations}

\begin{abstract}
BCS Bathocuproinedisulfonic acid disodium salt ESI-MS Electrospray ionisation mass spectrophotometry HEPES 4-(2-Hydroxyethyl)-1-piperazineethanesulfonic acid ROS Reactive oxygen species
\end{abstract}

\section{Acknowledgements}

This study was supported by the grant of Czech Science Foundation (project no. P303/12/G163) and by Charles University in Prague (grants no. $605712 \mathrm{C}$ and $1220314 \mathrm{~B}$; and project no. SVV $260064)$.

\section{References}

1 M. Daglia, Polyphenols as antimicrobial agents, Curr. Opin. Biotechnol., 2012, 23, 174-181.

2 S. Kumar and A. K. Pandey, Chemistry and biological activities of flavonoids: an overview, Sci. World J., 2013, 2013, 162750.

3 P. G. Pietta, Flavonoids as antioxidants, J. Nat. Prod., 2000, 63, 1035-1042.

4 P. Mladenka, L. Zatloukalova, T. Filipsky and R. Hrdina, Cardiovascular effects of flavonoids are not caused only by direct antioxidant activity, Free Radical Biol. Med., 2010, 49, 963-975.

5 J. E. Brown, H. Khodr, R. C. Hider and C. A. Rice-Evans, Structural dependence of flavonoid interactions with $\mathrm{Cu}^{2+}$ ions: implications for their antioxidant properties, Biochem. J., 1998, 330(Pt 3), 1173-1178.

6 H. El Hajji, E. Nkhili, V. Tomao and O. Dangles, Interactions of quercetin with iron and copper ions: complexation and autoxidation, Free Radical Res., 2006, 40, 303-320.

7 G. Escandar and L. Sala, Complexing behaviour of rutin and quercetin, Can. J. Chem., 1991, 69, 1994-2001.

8 M. T. Fernandez, M. L. Mira, M. H. Florencio and K. R. Jennings, Iron and copper chelation by flavonoids: an electrospray mass spectrometry study, J. Inorg. Biochem., 2002, 92, 105-111.

9 L. Mira, M. T. Fernandez, M. Santos, R. Rocha, M. H. Florencio and K. R. Jennings, Interactions of flavonoids with iron and copper ions: a mechanism for their antioxidant activity, Free Radical Res., 2002, 36, 1199-1208.

10 A. Pekal, M. Biesaga and K. Pyrzynska, Interaction of quercetin with copper ions: complexation, oxidation and reactivity towards radicals, BioMetals, 2011, 24, 41-49.

11 H. Tapiero, D. M. Townsend and K. D. Tew, Trace elements in human physiology and pathology. Copper, Biomed. Pharmacother., 2003, 57, 386-398.

$12 \mathrm{~K}$. Jomova and M. Valko, Advances in metal-induced oxidative stress and human disease, Toxicology, 2011, 283, 65-87.

13 A. Ala, A. P. Walker, K. Ashkan, J. S. Dooley and M. L. Schilsky, Wilson's disease, Lancet, 2007, 369, 397-408.

14 E. Berenshtein, B. Vaisman, C. Goldberg-Langerman, N. Kitrossky, A. M. Konijn and M. Chevion, Roles of ferritin and iron in ischemic preconditioning of the heart, Mol. Cell. Biochem., 2002, 234-235, 283-292.

15 G. Eskici and P. H. Axelsen, Copper and oxidative stress in the pathogenesis of Alzheimer's disease, Biochemistry, 2012, 51, 6289-6311.

16 S. A. Lowndes and A. L. Harris, The role of copper in tumour angiogenesis, J. Mammary Gland Biol. Neoplasia, 2005, 10, 299-310.

17 S. Rivera-Mancia, I. Perez-Neri, C. Rios, L. Tristan-Lopez, L. Rivera-Espinosa and S. Montes, The transition metals copper and iron in neurodegenerative diseases, Chem.Biol. Interact., 2010, 186, 184-199.

18 M. Riha, J. Karlickova, T. Filipsky, K. Macakova, R. Hrdina and P. Mladenka, Novel method for rapid copper chelation assessment confirmed low affinity of D-penicillamine for copper in comparison with trientine and 8hydroxyquinolines, J. Inorg. Biochem., 2013, 123, 80-87.

19 G. Righi, R. Antonioletti, I. P. Silvestri, N. D'Antona, D. Lambusta and P. Bovicelli, Convergent synthesis of mosloflavone, negletein and baicalein from crysin, Tetrahedron, 2010, 66, 1294-1298.

20 D. J. Sheskin, Handbook of Parametric and Nonparametric Statistical Procedures, Chapman \& Hall/CRC, London, 4th edn, 2007.

21 M. Guo, C. Perez, Y. Wei, E. Rapoza, G. Su, F. Bou-Abdallah and N. D. Chasteen, Iron-binding properties of plant phenolics and cranberry's bio-effects, Dalton Trans., 2007, 4951-4961.

22 P. Mladenka, K. Macakova, T. Filipsky, L. Zatloukalova, L. Jahodar, P. Bovicelli, I. P. Silvestri, R. Hrdina and L. Saso, In vitro analysis of iron chelating activity of flavonoids, J. Inorg. Biochem., 2011, 105, 693-701.

23 C. A. Perez, Y. Wei and M. Guo, Iron-binding and antiFenton properties of baicalein and baicalin, J. Inorg. Biochem., 2009, 103, 326-332.

24 J. Ren, S. Meng, C. E. Lekka and E. Kaxiras, Complexation of flavonoids with iron: structure and optical signatures, $J$. Phys. Chem. B, 2008, 112, 1845-1850. 
25 I. F. Cheng and K. Breen, On the ability of four flavonoids, baicilein, luteolin, naringenin, and quercetin, to suppress the Fenton reaction of the iron-ATP complex, BioMetals, 2000, 13, 77-83.

26 M. Melidou, K. Riganakos and D. Galaris, Protection against nuclear DNA damage offered by flavonoids in cells exposed to hydrogen peroxide: the role of iron chelation, Free Radical Biol. Med., 2005, 39, 1591-1600.

27 S. S. Park, I. Bae and Y. J. Lee, Flavonoids-induced accumulation of hypoxia-inducible factor (HIF)-1alpha/ 2alpha is mediated through chelation of iron, J. Cell. Biochem., 2008, 103, 1989-1998.

28 P. Sestili, A. Guidarelli, M. Dacha and O. Cantoni, Quercetin prevents DNA single strand breakage and cytotoxicity caused by tert-butylhydroperoxide: free radical scavenging versus iron chelating mechanism, Free Radical Biol. Med., 1998, 25, 196-200.

29 S. A. van Acker, G. P. van Balen, D. J. van den Berg, A. Bast and W. J. van der Vijgh, Influence of iron chelation on the antioxidant activity of flavonoids, Biochem. Pharmacol., 1998, 56, 935-943.

30 S. A. van Acker, D. J. van den Berg, M. N. Tromp, D. H. Griffioen, W. P. van Bennekom, W. J. van der Vijgh and A. Bast, Structural aspects of antioxidant activity of flavonoids, Free Radical Biol. Med., 1996, 20, 331-342.

31 J. P. Cornard and J. C. Merlin, Comparison of the chelating power of hydroxyflavones, J. Mol. Struct., 2003, 651, 381-387.

32 T. Filipsky, M. Riha, R. Hrdina, K. Vavrova and P. Mladenka, Mathematical calculations of iron complex stoichiometry by direct UV-Vis spectrophotometry, Bioorg. Chem., 2013, 49, 18.

$33 \mathrm{P}$. Job, Recherches sur la formation de complexes mineraux en solution, et sur leur stabilite, Ann. Chim., 1928, 9, 113134.

34 C. A. Rice-Evans, N. J. Miller and G. Paganga, Structureantioxidant activity relationships of flavonoids and phenolic acids, Free Radical Biol. Med., 1996, 20, 933-956.

35 M. M. Silva, M. R. Santos, G. Caroco, R. Rocha, G. Justino and L. Mira, Structure-antioxidant activity relationships of flavonoids: a re-examination, Free Radical Res., 2002, 36, 1219-1227.

36 J. M. Herrero-Martinez, M. Sanmartin, M. Roses, E. Bosch and C. Rafols, Determination of dissociation constants of flavonoids by capillary electrophoresis, Electrophoresis, 2005, 26, 1886-1895.
37 R. de Souza, E. Sussuchi and W. De Giovani, Synthesis, Electrochemical, Spectral, and Antioxidant Properties of Complexes of Flavonoids with Metal Ions, Synth. React. Inorg. Met.-Org. Chem., 2003, 33, 1125-1144.

38 S. Teixeira, C. Siquet, C. Alves, I. Boal, M. P. Marques, F. Borges, J. L. Lima and S. Reis, Structure-property studies on the antioxidant activity of flavonoids present in diet, Free Radical Biol. Med., 2005, 39, 1099-1108.

39 S. A. van Acker, M. J. de Groot, D. J. van den Berg, M. N. Tromp, G. Donne-Op den Kelder, W. J. van der Vijgh and A. Bast, A quantum chemical explanation of the antioxidant activity of flavonoids, Chem. Res. Toxicol., 1996, 9, 1305-1312.

40 E. Lekka Ch, J. Ren, S. Meng and E. Kaxiras, Structural, electronic, and optical properties of representative $\mathrm{Cu}$ flavonoid complexes, J. Phys. Chem. B, 2009, 113, 6478-6483.

41 B. Sarkar, A. Sass-Kortsak, R. Clarke, S. H. Laurie and P. Wei, A comparative study of in vitro and in vivo interaction of $\mathrm{D}$ penicillamine and triethylenetetramine with copper, Proc. $R$. Soc. Med., 1977, 70(suppl. 3), 13-18.

42 D. Del Rio, A. Rodriguez-Mateos, J. P. Spencer, M. Tognolini, G. Borges and A. Crozier, Dietary (poly)phenolics in human health: structures, bioavailability, and evidence of protective effects against chronic diseases, Antioxid. Redox Signaling, 2013, 18, 1818-1892.

43 J. F. Collins, J. R. Prohaska and M. D. Knutson, Metabolic crossroads of iron and copper, Nutr. Rev., 2010, 68, 133-147.

44 J. M. Calderon-Montano, E. Burgos-Moron, C. PerezGuerrero and M. Lopez-Lazaro, A review on the dietary flavonoid kaempferol, Mini-Rev. Med. Chem., 2011, 11, 298344.

45 I. R. Record, J. K. McInerney and I. E. Dreosti, Black tea, green tea, and tea polyphenols. Effects on trace element status in weanling rats, Biol. Trace Elem. Res., 1996, 53, 2743.

46 C. Coudray, C. Bousset, J. C. Tressol, D. Pepin and Y. Rayssiguier, Short-term ingestion of chlorogenic or caffeic acids decreases zinc but not copper absorption in rats, utilization of stable isotopes and inductively-coupled plasma mass spectrometry technique, Br. J. Nutr., 1998, 80, 575-584.

47 Z. Gao, H. Xu, X. Chen and H. Chen, Antioxidant status and mineral contents in tissues of rutin and baicalin fed rats, Life Sci., 2003, 73, 1599-1607. 